\title{
Genetic predisposition to infection in a case of atypical hemolytic uremic syndrome (aHUS)
}

Lambertus van den Heuvel ${ }^{1,2,3 *}$, Kristian Riesbeck ${ }^{4 *}$, Omaima El Tahir ${ }^{5,6}$, Valentina Gracchi ${ }^{7}$, Mariann Kremlitzka ${ }^{8}$, Servaas Morre ${ }^{5,9}$, Marceline van Furth ${ }^{6}$, Birendra Singh ${ }^{4}$, Marcin Okroj $^{10}$, Nicole van de $\operatorname{Kar}^{1}$, Anna M. Blom ${ }^{8 *}$, Elena Volokhina ${ }^{1,2^{\star}}$

${ }^{*}$ Contributed equally

${ }^{1}$ Department of Pediatric Nephrology, Radboud university medical center, Nijmegen, The Netherlands; ${ }^{2}$ Department of Laboratory Medicine, Radboud university medical center, Nijmegen, The Netherlands; ${ }^{3}$ Department of Pediatrics, University Hospitals Leuven, Belgium; ${ }^{4}$ Clinical Microbiology, Department of Translational Medicine, Lund University, Malmö, Sweden; ${ }^{5}$ Department of Medical Microbiology and Infection Control, Laboratory of Immunogenetics VU University Medical Center, Amsterdam, The Netherlands; ${ }^{6}$ Department of Pediatric Infectious Diseases, VU University medical center, Amsterdam, The Netherlands; ${ }^{7}$ Department of Pediatric Nephrology, University of Groningen, University Medical Centre Groningen, The Netherlands; ${ }^{8}$ Medical Protein Chemistry, Department of Translational Medicine, Lund University, Malmö, Sweden; ${ }^{9}$ Department of Genetica and Cell Biology, Institute for Public Health Genomics (IPHG), Research School GROW (School for Oncology \& Developmental Biology), Faculty of Health, Medicine \& Life Sciences, University of Maastricht, The Netherlands; ${ }^{10}$ Department of Medical Biotechnology, Intercollegiate Faculty of Biotechnology UG-MUG, Medical University of Gdańsk, Poland

Running title: Genetic predisposition to infection in aHUS

\section{Corresponding author:}

Dr. E. Volokhina

Department of Pediatric Nephrology (830), Radboud university medical center

P.O. Box 9101, 6500 HB Nijmegen, The Netherlands

Tel: +31-24-3616353

Email: Elena.Volokhina@radboudumc.nl

This is author's version of the article.

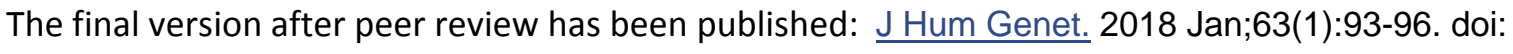
10.1038/s10038-017-0356-0. Epub 2017 Nov 13. 


\section{Abstract}

Genetic defects causing uncontrolled complement activation are associated with aHUS. NonEHEC infections can trigger the disease, however, complement defects predisposing to such infections have not yet been studied.

We describe a two-month old patient infected with different Gram-negative bacterial species resulting in aHUS. Serum analysis revealed slow complement activation kinetics. Rare variant R229C was found in complement inhibitor vitronectin. Recombinant mutated vitronectin showed enhanced complement inhibition in vitro and may have been a predisposing factor for infection.

Our work indicates that genetic changes in aHUS can not only result in uncontrolled complement activation but also increase vulnerability to infections contributing to aHUS.

Key words: atypical HUS, complement inhibition, infection, mutation, vitronectin 


\section{Introduction}

Hemolytic uremic syndrome (HUS) is a devastating renal disease, which is characterized by hemolytic anemia, thrombocytopenia and acute renal failure. Most HUS cases are caused by infection with enterohemorrhagic Escherichia coli (EHEC). However, 5-10\% of HUS patients have a more severe atypical form (aHUS). Overactive complement is considered to be a central element in aHUS pathogenesis ${ }^{1}$.

The complement system, a part of the innate immune system, can be activated via three pathways: the classical, the lectin and the alternative. These pathways converge at the cleavage and activation of the central complement component C3 leading further to formation of the terminal C5b-9 complement complex (TCC) and release of the potent anaphylatoxins $\mathrm{C} 3 \mathrm{a}$ and $\mathrm{C} 5 \mathrm{a}^{1}$.

Sequence variants that lead to impaired complement regulation of C3 activity in aHUS are found in genes encoding $\mathrm{CFH}, \mathrm{CFI}, \mathrm{MCP}, \mathrm{C} 3, \mathrm{CFB}$ and thrombomodulin. Moreover, important $\mathrm{C} 3$ convertase inhibitor $\mathrm{CFH}$ can be affected by autoantibodies (anti-CFH) ${ }^{1}$. Next to these abnormalities,

aHUS episodes can sometimes be triggered by non-EHEC infections, including Streptococcus pneumoniae, Pseudomonas aeruginosa, influenza A, HIV and others ${ }^{1,2}$. Several cases of infection with Bordetella pertussis have also been reported ${ }^{3-5}$. The infections trigger initial complement attack, which cannot be adequently controlled due to dysregulating genetic changes and may cause renal damage in aHUS. Nevertheless, genetic predisposition to infections in aHUS has not yet been studied. 


\section{Case report}

A female infant was diagnosed with whooping cough at the age of seven weeks. The presence of Bordetella pertussis infection was confirmed by serological analysis, where IgG values increased from 1 Virotech unit (VE)/mL to $10 \mathrm{VE} / \mathrm{mL}$ in first eight days of the disease. At day 10 after the onset of infection, the patient developed acute renal failure (serum creatinine $257 \mu \mathrm{mol} / \mathrm{L}$, urea $27.5 \mathrm{mmol} / \mathrm{L}$ ), hemolytic anemia (Hb $2.8 \mathrm{mmol} / \mathrm{L}, \mathrm{LDH} 3591 \mathrm{U} / \mathrm{L}$ ) and mild thrombocytopenia (platelets 147*109/L) and was consequently diagnosed with aHUS. Infection with EHEC 0157 was excluded by fecal culture and PCR. ADAMTS13 activity was normal (53\%), which excluded thrombotic thrombocytopenic purpura. At this time the patient also developed pneumonia (sputum positive for Moraxella catarrhalis) and sepsis (Klebsiella oxytoca in blood). The infant received blood transfusions, continuous venovenous hemofiltration (CVVH) and was venilated. She recovered completely from the aHUS 10 days later (20 days after the onset of the pertussis) with normalized renal function (serum creatinine 43 $\mu \mathrm{mol} / \mathrm{L}$, urea $1 \mathrm{mmol} / \mathrm{L})$. During a 10 year follow-up, patient presented no other episode of aHUS or severe infection.

Due to infections with Gram-negative bacteria, we analyzed activity of the three complement pathways after the patient had recovered from aHUS. These were within the normal range (Table 1). The in vitro complement activation of patient serum was compared to that of normal human serum (NHS). In patient's serum C3 activation rate (expressed as generation of C3bc) was comparable to the rate in NHS, but the TCC generation was delayed (Figure $1 \mathrm{~A}$ and $\mathrm{B}$ ).

Genetic screening of the alternative pathway indicated presence of a heterozygous missense variant in thrombomodulin (A43T), which was previously described as pathogenic in aHUS ${ }^{6}$. No other changes in alternative pathway or anti-CFH autoantibodies were detected.

Because kinetic experiments have shown decreased rate of TCC generation, we analyzed the patient for possible defects in genes encoding TCC components and TCC inhibitors (vitronectin, clusterin and CD59). A heterozygous variant rs782409757:c.685C>T (R229C) was found in gene encoding vitronectin. In silico analysis indicated this variant as deleterious (SIFT score 0.0) and probably damaging (PolyPhen-2 score 1.0) as a large positively charged amino acid is replaced by an unpaired cysteine. Patient vitronectin plasma levels were normal (Table 1). In vitro experiments using 
purified recombinant proteins revealed that mutant vitronectin was more potent in complement inhibition compared to the wild type (Figure 1C).

The $\mathrm{c} .685 \mathrm{C}>\mathrm{T}(\mathrm{R} 229 \mathrm{C})$ is a rare variant, reported with the frequency of $0.0015 \%$ in European population (http://exac.broadinstitute.org/variant/17-26696034-G-A). Moreover, we tested the variant in 390 Dutch children who survived bacterial meningitis, since variation in innate immune response genes also affects susceptibility to meningitis ${ }^{7}$. None of the children had the vitronectin variant, which may be explained by low incidence of the change. 


\section{Discussion}

In this work we presented a case of aHUS in a two-month old infant. Although B. pertussis aHUS cases have been described before, to our knowledge, this is the first case associated with simultaneous isolation of $K$. oxytoca and $M$. catarrhalis.

Functional assessment of the complement system did not reveal major abnormalities (Table 1). Low functional activity of MBL-mediated lectin pathway was found, which is very common in the human population thus not considered as defect. In our previous work we have found that MBL deficiency is not more common in aHUS patients than in healthy controls ${ }^{8}$.

The rate of TCC formation in patient's serum was slower than in the NHS. This is in line with less efficient lysis of sheep erythrocytes by the TCC complex, assembled from purified components in the presence of recombinant vitronectin with R229C mutation. Delayed TCC response may have caused inefficient initial clearance of Gram-negative bacteria in the patient, which resulted in infection and profound complement activation. Due to the presence of thrombomodulin change, complement attack was poorly contolled at the level of C3 activation with aHUS episode as a result.

Importantly, aHUS patients are currently treated with TCC blocker eculizumab. Patients that carry TCC inhibiting variants, as for the fitst time described here, may require lower drug doses.

Taken together, our work indicates that a genetic cause may contribute to aHUS not only by a well-known effect of complement dysregulation, but also by enhancing vulnerability to infections during early infancy. 


\section{Aknowledgements}

This work was supported by the grants from the Dutch Kidney Foundation (13OI116, KFB 11.007, IP 10.22), European Renal Association - European Dialysis and Transplantation Association (ERA STF 138-2013, ERA LTF 203-2014), and European Society for Pediatric Nephrology (2014.03).

\section{Conflict of interest}

Dr. N.C.A.J. van de Kar is a member of the international advisory board of Alexion. Other authors declare no conflict of interest. 


\section{References}

1. Westra, D., Wetzels, J., Volokhina, E., van den Heuvel, L. \& van de Kar, N. A new era in the diagnosis and treatment of atypical haemolytic uraemic syndrome. Neth. J. Med. 70, 121-129 (2012).

2. Karpman, D., Loos, S., Tati, R. \& Arvidsson, I. Haemolytic uraemic syndrome. Journal of internal medicine. J. Intern. Med. 281, 123-148 (2017).

3. Obando, I., Camacho, M., Falcon-Neyra, D., Hurtado-Mingo, A. \& Neth, O. Atypical hemolytic uremic syndrome associated with Bordetella pertussis infection. Pediatr. Infect. Dis. J. 31, 1210 (2012).

4. Chaturvedi, S., Licht, C. \& Langlois, V. Hemolytic uremic syndrome caused by Bordetella pertussis infection. Pediatr. Nephrol. 25, 1361-1364 (2010).

5. Pela, I., Seracini, D., Caprioli, A., Castelletti, F. \& Giammanco, A. Hemolytic uremic syndrome in an infant following Bordetella pertussis infection. Eur. J. Clin. Microbiol. Infect. Dis. 25, 515-517 (2006).

6. Delvaeye, M., Noris, M., De Vriese, A., Esmon, C., Esmon, N., Ferrell, G. et al. Thrombomodulin mutations in atypical hemolytic-uremic syndrome. N. Engl. J. Med. 361, 345-357 (2009).

7. van Well, G., Sanders, M., Ouburg, S., Kumar, V., van Furth, A. \& Morre, S. Single nucleotide polymorphisms in pathogen recognition receptor genes are associated with susceptibility to meningococcal meningitis in a pediatric cohort. PloS One. 8, e64252. doi:

10.1371/journal.pone.0064252 (2013).

8. Volokhina, E., Westra, D., van der Velden, T., van de Kar, N., Mollnes, T. \& van den Heuvel,

L. Complement activation patterns in atypical haemolytic uraemic syndrome during acute phase and in remission. Clin. Exp. Immunol. 181, 306-313 (2015).

9. Bergseth, G., Ludviksen, J., Kirschfink, M., Giclas, P., Nilsson, B. \& Mollnes. T. An international serum standard for application in assays to detect human complement activation products. Mol. Immunol. 56, 232-239 (2013). 
10. Escudero-Esparza. A., Kalchishkova. N., Kurbasic. E., Jiang. W. \& Blom A. The novel complement inhibitor human CUB and Sushi multiple domains 1 (CSMD1) protein promotes factor Imediated degradation of $\mathrm{C} 4 \mathrm{~b}$ and $\mathrm{C} 3 \mathrm{~b}$ and inhibits the membrane attack complex assembly. FASEB J. 27, 5083-5093 (2013).

11. Singh, B., Blom, A., Unal, C., Nilson, B., Morgelin, M. \& Riesbeck, K. Vitronectin binds to the head region of Moraxella catarrhalis ubiquitous surface protein A2 and confers complement-inhibitory activity. Mol. Microbiol. 75, 1426-1444 (2010). 
Table 1. Complement activity assessment in aHUS patient.

\begin{tabular}{lll}
\hline Complement pathway & Patient's value & Reference range \\
\hline Classical pathway $(\%)^{1}$ & 77.4 & $69-129$ \\
Alternative pathway $(\%)^{1}$ & 75.1 & $30-113$ \\
Lectin pathway (MBL-mediated) $(\%)^{1}$ & 18.2 & $0-125$ \\
& & \\
Plasma vitronectin concentration $(\mu \mathrm{g} / \mathrm{mL})^{2}$ & 190 & $185-595^{4}(286, \mathrm{n}=20)^{3}$ \\
& & \\
\hline
\end{tabular}

${ }^{1}$ Measured using Wieslab® Complement system Screenkit (Euro Diagnostica) and given as percentage of the positive control, provided with the assay. Reference range is presented as indicated by the manufacturer.

${ }^{2}$ Measured using Human Vitronectin Total ELISA Kit (Innovative Research).

${ }^{3}$ Reference range was determined as range of values, measured in a healthy control group, median value and number of analyzed controls are indicated in parenthesis. 


\section{Figure legends}

Figure 1. A. Serum of patient carrying R229C mutation in vitronectin and normal human serum pool (NHS) were incubated at $37^{\circ} \mathrm{C}$ with gentle agitation. Samples were collected at $0,10,20,30$ and 60 minutes of incubation and C3bc levels were quantified in complement activation units per $\mathrm{mL}$ $(\mathrm{CAU} / \mathrm{mL})$ using international complement standard $\# 2^{9}$. Data were collected in three independent experiments and presented as mean \pm standard error. B. TCC levels were quantified in the same experimental set up as $\mathrm{C} \mathrm{bc}^{9}$. C TCC was allowed to form on the surface of sheep erythrocytes from purified components of (C5b6, C7, C8, C9) as previously described ${ }^{10}$ in the presence of various concentrations of purified recombinant vitronectin variants. The wild type (rVTN wt) and mutant (rVTN R229C) vitronectin variants (amino acids 20-396) were produced in HEK293T cells as previously described ${ }^{11}$. Efficiency of TCC formation was quantified as percentages of lysed erythrocytes. Data were collected in four independent experiments and presented as mean+ standard error. 
